\title{
Rapid distinction of intracellular and extracellular proteins using NMR diffusion measurements
}

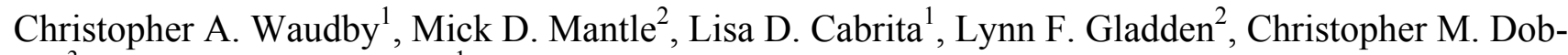 \\ son $^{3}$ and John Christodoulou ${ }^{1, *}$ \\ ${ }^{1}$ Institute of Structural and Molecular Biology, University College London and Birkbeck College, Gower Street, London \\ WC1E 6BT, UK \\ ${ }^{2}$ Department of Chemical Engineering and Biotechnology, University of Cambridge, Pembroke Street, Cambridge CB2 3RA, \\ UK \\ ${ }^{3}$ Department of Chemistry, University of Cambridge, Lensfield Road, Cambridge CB2 1EW, UK
}

Supporting Information Placeholder

\begin{abstract}
In-cell NMR spectroscopy offers a unique opportunity to begin to investigate the structures, dynamics and interactions of molecules within their functional environments. An essential aspect of this technique is to define whether or not observed signals are attributable to intracellular species rather than components of the extracellular medium. We report here the results of NMR measurements of the diffusion behavior of proteins expressed within bacterial cells, and find that these experiments provide a rapid and non-destructive probe of localization within cells and can be used to determine the size of the confining compartment. We show that diffusion can also be exploited as an editing method to eliminate extracellular species from high-resolution multi-dimensional spectra, and should be applicable to a wide range of problems. This approach is demonstrated here for a number of protein systems, using both ${ }^{15} \mathrm{~N}$ and ${ }^{13} \mathrm{C}$ (methyl-TROSY) based acquisition.
\end{abstract}

NMR spectroscopy, in conjunction with specific isotope labeling methods, is a powerful approach for the direct observation of molecules such as proteins within their natural and highly complex cellular environments, as well as in the dilute solutions commonly studied under laboratory conditions. In recent years, for example, a number of studies have succeeded in characterizing at the atomic level the structures and properties of proteins within cells ${ }^{1,2}$. A fundamental requirement of all in-cell NMR studies is to determine unequivocally whether or not specific resonances in a given spectrum originate from species that are localized within the cell rather than in the extracellular medium. As in most cases the chemical shifts of intracellular and extracellular species are likely to be very similar, this exercise is in general non-trivial; indeed, the most common approach at present is simply to centrifuge the sample and to compare spectra of the supernatant with those from the original sample ${ }^{3}$. A non-destructive alternative is highly desirable, and we report here a simple approach based on pulsed-field gradient NMR diffusion measurements that can provide the required information in a very short time, typically less than two minutes.

NMR spectroscopy using pulsed-field gradients provides a powerful method for the characterization of molecular diffu- sion $^{4}$, and for example has been applied to study the diffusion of proteins within cell lysates and other crowded solutions in an effort to develop an understanding of the physical processes controlling macromolecular transport within the cell ${ }^{5}$. Diffusion may also be used as a means of spectral editing, e.g. to suppress the resonances of small molecule metabolites from spectra of macromolecules within cell lysates ${ }^{6}$. However, within intact cells the measurement of protein diffusion has been dominated by a range of fluorescence methods ${ }^{7,8}$. Such measurements have demonstrated that the effective viscosity of the eukaryotic cytosol is typically 3-4 times greater than that of water ${ }^{9}$, while in the highly crowded bacterial cytosol, containing $300-400 \mathrm{mg} \mathrm{mL}^{-1}$ of macromolecules ${ }^{10}$, translational diffusion of proteins is reduced by approximately an order of magnitude relative to dilute aqueous solution ${ }^{11}$.

An essential difference between in-cell studies and conventional solution studies is that the diffusion of any molecule under observation is restricted to a much smaller volume, by being confined within a cell or a cellular compartment. In the specific case of $E$. coli, the system studied in this paper, the total cellular volume corresponds to approximately $0.5 \mathrm{fL}$. On the timescale of NMR diffusion measurements, the consequence of this confinement will be a reduction in the apparent diffusion coefficient, suggesting that diffusion-edited NMR experiments could be used to distinguish between intracellular and extracellular species.

In this communication, we first explore this approach using the intrinsically disordered protein $\alpha$-synuclein ( $\alpha$ Syn), which has previously been reported to give well-resolved NMR resonances when expressed within $E$. coli cells ${ }^{12} \cdot{ }^{15} \mathrm{~N}$-edited stimulated-echo (STE) diffusion experiments ${ }^{13}$ of such samples were recorded here using a long diffusion delay, $\Delta$, of $300 \mathrm{~ms}$, in order to maximize the contrast between slowly and rapidly diffusing species. The signal intensities $I(G)$ for a sample of cells expressing $\alpha$ Syn, and for purified $\alpha$ Syn added separately to the extracellular medium of a sample of cells, are plotted in Fig. 1A, and are fitted to the Stejskal-Tanner equation ${ }^{14}$ to determine the apparent diffusion coefficient, $D_{\text {app }}$ :

$$
I(G)=I(0) \exp \left[-\gamma^{2} G^{2} \delta^{2} s^{2}(\Delta-\delta / 3-\tau / 2) D_{\text {app }}\right]
$$

where $I(0)$ is the signal intensity in the absence of gradients, $\gamma$ is the magnetogyric ratio of the proton, $\delta$ is the gradient pulse 
length, $s$ is the gradient shape factor, $\tau$ is the delay between bipolar gradients, and $G$ is the gradient strength.
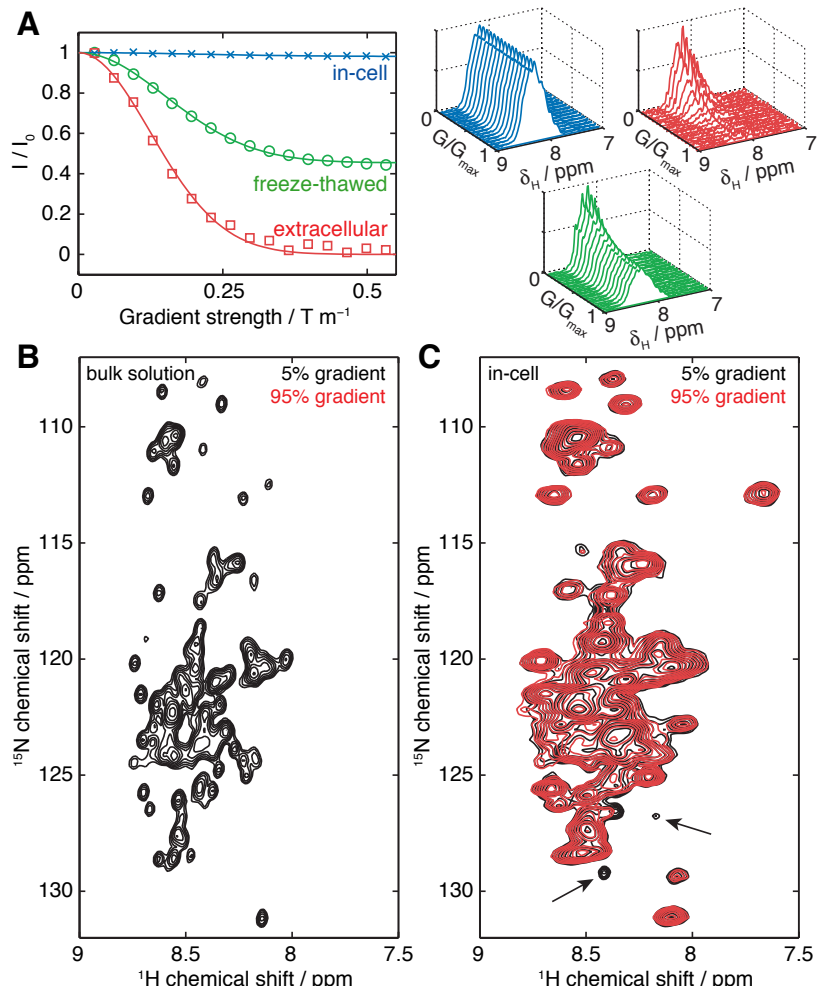

Figure 1. (A) $300 \mathrm{~ms}{ }^{15} \mathrm{~N}$-edited XSTE diffusion NMR measurements ${ }^{13}$ of cells in which $\alpha$ Syn has been expressed (blue), cells partially lysed following freeze-thawing with liquid $\mathrm{N}_{2}$ (green), and purified $\alpha$ Syn added to the extracellular medium of a sample of cells (red). (B, C) Diffusion-edited (300 ms XSTE) HSQC NMR spectra ${ }^{15}$ of (B) purified $\alpha$ Syn in bulk solution, and (C) cells expressing $\alpha$ Syn. The gradient strength used in each experiment is indicated relative to the maximum accessible gradient strength of $0.55 \mathrm{~T} \mathrm{~m}^{-1}$. Arrows highlight resonances of extracellular species that are absent at higher gradient strengths.

The intensity of the resonances of cell samples at the maximum gradient strength applied here, $G=0.55 \mathrm{~T} \mathrm{~m}^{-1}$, was observed to be $97 \pm 1 \%$ of that in the absence of the gradient, with a fitted value of $D_{\text {app }}=4.6 \pm 0.8 \times 10^{-13} \mathrm{~m}^{2} \mathrm{~s}^{-1}$. By contrast, resonances from exogenous $\alpha$ Syn are effectively completely attenuated at this maximum gradient strength. The measured value of $D=(1.2 \pm 0.1) \times 10^{-10} \mathrm{~m}^{2} \mathrm{~s}^{-1}$ is only slightly reduced relative to that observed for $\alpha$ Syn in bulk solution, $D$ $=(1.3 \pm 0.1) \times 10^{-10} \mathrm{~m}^{2} \mathrm{~s}^{-1}$, which is itself comparable to values from previous measurements and corresponds to a hydrodynamic radius of $25 \pm 2 \AA^{16}$. This result reveals that intracellular and extracellular protein molecules can be distinguished clearly as their apparent diffusion coefficients differ by almost three orders of magnitude. Each experiment in the present study was acquired within six minutes, and this time could be reduced to under a minute by omitting intermediate gradient points.

The large difference in the apparent diffusion coefficients for a protein inside and outside the cell provides an opportunity to use pulsed-field gradients to dephase selectively the resonances of rapidly diffusing extracellular species and so to use diffusion-edited experiments to observe intracellular species exclusively. This strategy is illustrated in Fig. 1B,C, where diffusion-edited HSQC spectra ${ }^{15}$ have been recorded of purified $\alpha$ Syn in bulk solution (Fig. 1B) and of $\alpha$ Syn expressed within E. coli cells (Fig. 1C). The sensitivity of these measurements was found to be approximately $40 \%$ of that of a conventional HSQC experiment, due in part to an increase in relaxation that occurs during the longer pulse sequence, but predominantly to the $50 \%$ loss of signal inherent in all stimulated echo experiments, as only a single transverse magnetization component can be stored longitudinally during the diffusion delay.

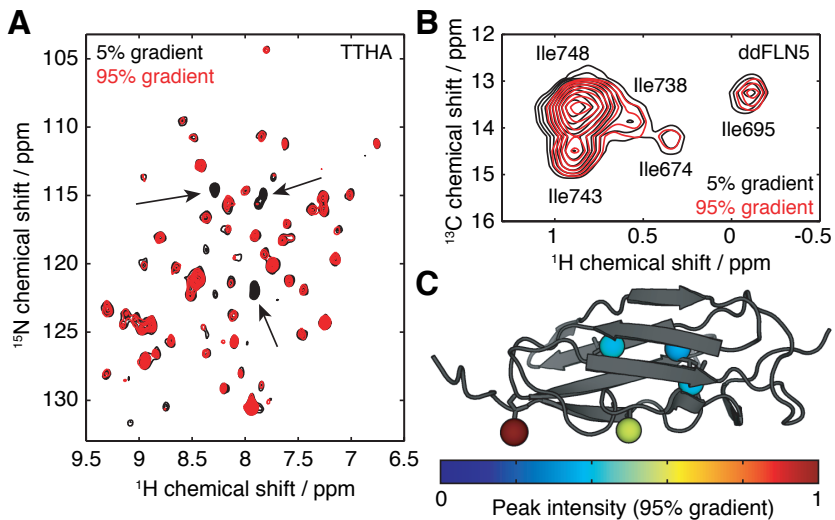

Figure 2. (A) Diffusion-edited (300 ms ${ }^{15} \mathrm{~N}$ XSTE) HSQC NMR spectra of TTHA expressed within cells. The gradient strength used in each experiment is indicated relative to the maximum accessible gradient strength of $0.55 \mathrm{~T} \mathrm{~m}^{-1}$. Arrows highlight resonances of extracellular species that are absent at higher gradient strengths. (B) Diffusion-edited (300 ms STE) ${ }^{13} \mathrm{C}$ HMQC (methyl-TROSY) NMR spectra of $\left[\mathrm{Ile}-{ }^{13} \mathrm{CH}_{3}\right]$-ddFLN5 expressed within cells. (C) Crystal structure of ddFLN5 (pdb 1QFH) showing isoleucine $\mathrm{C} \delta$ groups, colored according to the peak intensity observed within the cell.

To validate further this approach, additional in-cell samples were prepared and subjected to partial lysis induced by freezethawing with liquid $\mathrm{N}_{2}$. The maximum attenuation of the stimulated echo was observed to be $55 \pm 2 \%$ (Fig. 1A), indicating that approximately half of the protein had escaped from the cell. This value compares to $51 \pm 1 \%$ determined from analysis of the supernatant following centrifugation and shows the NMR method to be highly robust, at least for the bacterial cells studied in this communication.

We have also investigated the application of diffusionedited methods to the observation of two representative folded proteins expressed within the cell. TTHA1718 ('TTHA') is a 66-residue folded protein that has been shown to give wellresolved HSQC spectra when expressed within the cell ${ }^{2}$. We find here that well-resolved diffusion-edited HSQC spectra may also be acquired for such samples (Fig. 2A). As was the case for $\alpha$ Syn, a number of resonances are absent at higher gradient strengths and therefore can be attributed to extracellular species (Fig. 2A, arrows). However, in our hands we also found that TTHA samples showed higher levels of leakage than was typically observed for $\alpha$ Syn: the signal intensity at the maximum gradient strength was $81 \pm 3 \%$ of that in the absence of the gradient, indicating that approximately $19 \%$ of the protein was present outside the cell in this experiment. In such circumstances, diffusion-edited acquisition is particularly valuable in permitting the exclusive observation of intracellular species. 
The second folded protein we examined is ddFLN5, a 113residue immunoglobulin domain from the Dictyostelium discoideum gelation factor ABP-120, currently under study within our group as a ribosome-nascent chain complex for the investigation of cotranslational folding ${ }^{17,18}$. However, in contrast to the smaller TTHA molecule, resonances of ddFLN5 could not be observed in ${ }^{1} \mathrm{H}^{-15} \mathrm{~N}$ HSQC spectra of cell samples (Fig. $\mathrm{S} 1$ ), indicating the presence of rapid transverse relaxation that may result from the viscosity of the cytosol, and also potentially from specific or non-specific interactions with other components of the cell ${ }^{19}$. We therefore prepared deuterated cell samples with selective ${ }^{13} \mathrm{CH}_{3}$ labeling of isoleucine $\mathrm{C} \delta$ methyl groups, in order to utilize the methyl-TROSY effect to reduce the effect of transverse relaxation ${ }^{20}$. Methyl-TROSY methods were also combined with STE diffusion measurements ${ }^{21}$ which, as illustrated for $\alpha$ Syn, may be acquired rapidly in a $1 \mathrm{D}$ manner to measure echo attenuations. In addition, diffusion-edited ${ }^{1} \mathrm{H}_{-}{ }^{13} \mathrm{C}$ HMQC spectra were acquired, and are shown in Fig. 2B for a sample of cells in which isoleucinelabeled ddFLN5 has been expressed. All ddFLN5 isoleucine resonances were observed, with identical chemical shifts as observed for the protein in bulk solution (Fig. S2) indicating that no major structural changes have occurred within the cell. Peak intensities at the maximum gradient strength were $83 \pm 2$ $\%$ of those in the absence of the gradient, indicating that approximately $17 \%$ of the protein was present outside of the cell.

Significant residue-to-residue variation was observed in the intensities of resonances in the diffusion-edited spectrum of ddFLN5 (Fig. 2B). By contrast, intensities in the spectrum of the protein in bulk solution are close to uniform (Fig. S2). To investigate this broadening effect further, the relative peak intensities were projected onto the structure of the protein (Fig. 2C), from which it may be observed that the greatest intensities in the diffusion-edited spectrum are found for residues on the surface of the protein. The intensities are inversely correlated with predicted methyl $S^{2}$ order parameters ${ }^{22}\left(r^{2}=\right.$ 0.90 , Fig. S3) and, given the slow rotational diffusion within the crowded cytosol, this suggests that the extent of local mobility on ps-ns timescales may be an important factor in determining the observability of intracellular material. However, interactions between the protonated methyl groups within the core of the protein might also contribute significantly to the observed relaxation. Future work, combining diffusion-edited acquisition with spin relaxation measurements, has the potential to enable these effects to be interpreted in more detail.

The apparent diffusion coefficient determined by NMR measurements is related to the mean square displacement during the diffusion delay, $D_{\text {app }}=\left\langle Z^{2}\right\rangle / 2 \Delta$. For an intracellular species, $<Z^{2}>$ is limited by the size of the cell, and the apparent diffusion coefficient may therefore provide information on the size of the confining compartment. To investigate the possibility of obtaining such information, in a further series of experiments we recorded a series of diffusion measurements using a probe (Bruker Diff30) that is able to provide a gradient strength of up to $11.7 \mathrm{~T} \mathrm{~m}^{-1}$.

This approach provided larger echo attenuations, allowing the determination of the apparent diffusion coefficients of intracellular $\alpha$ Syn. ${ }^{1} \mathrm{H}$ spin-echo experiments were recorded for four values of $\Delta$ from 11 to $20 \mathrm{~ms}$, and echo attenuations of up to $90 \%$ were observed in all cases (Fig. 3). These attenuations were independent of $\Delta$, indicating that diffusion is restricted, and fitting the data to Eq. 1 indicates RMS displace- ments of $0.33 \pm 0.02 \mu \mathrm{m}$ (Fig. 3, inset). This is comparable in magnitude to an RMS displacement of $0.47 \mu \mathrm{m}$ estimated from Brownian dynamics simulations ${ }^{23}$ for a compartment size of $0.5 \times 0.5 \times 2 \mu \mathrm{m}$, typical for an $E$. coli cell. Thus, the characterization of restricted diffusion provides not only the ability to quantify intracellular localization, but also to provide an estimate of the size of the confining compartment. We note that we do not observe diffusion-diffraction effects in these measurements, for example, as previously observed for water diffusing in red blood cells ${ }^{24}$. This finding may reflect a combination of heterogeneity in the cell size and lack of alignment within the sample, and spatial averaging occurring during the finite gradient pulse length, resulting in shallower diffraction minima ${ }^{25}$.

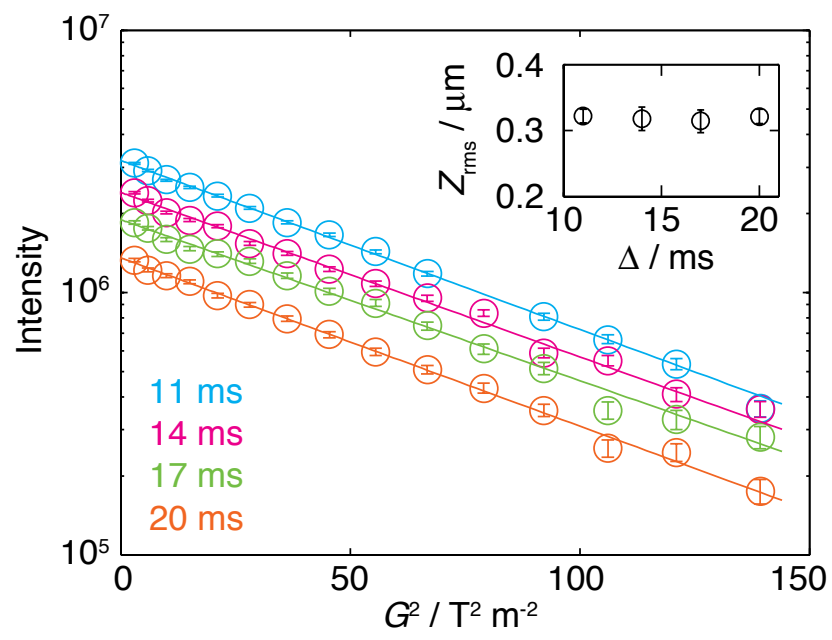

Figure $3 .{ }^{1} \mathrm{H}$ spin-echo measurements $(\delta=2 \mathrm{~ms})$ of an in-cell sample of $\alpha$ Syn, with the diffusion time, $\Delta$, varied between 11 and 20 $\mathrm{ms}$ as indicated. Data are fitted to Eq. 1, and (inset) the RMS displacements, $Z_{\mathrm{rms}}=\left(2 D_{\mathrm{app}} \Delta\right)^{1 / 2}$, are plotted as a function of $\Delta$.

Finally, we consider the role of diffusion measurements in the study of other types of cells. The diffusion-editing methodology described in this communication is particularly effective due to the effect of restricted dimensions on the apparent diffusion coefficient. However, the magnitude of this enhancement decreases when the cell size becomes comparable to the RMS displacement occurring during the diffusion measurement, and simple estimates suggest that the effects described here for $E$. coli cells are not likely to be significant at length scales of $10 \mu \mathrm{m}$ and above - e.g. for mammalian cells and particularly for $X$. laevis oocytes used previously for incell NMR studies ${ }^{26,27}$. However, it should remain possible to distinguish intracellular and extracellular species via the slower diffusion in the cytoplasm. More importantly, restricted diffusion is likely to be a key probe for observing the confinement of species to compartments or organelles within the cell.

In summary, diffusion measurements are a powerful component of the biomolecular NMR toolkit, with diverse applications including the study of protein aggregation ${ }^{28,29}$ and large macromolecular complexes such as the ribosome and ribosome nascent chain complexes ${ }^{17,30}$. We have shown in this communication that diffusion experiments also provide a means of rigorously identifying and selectively observing protein molecules within the living cell. We believe that such 
methods are widely applicable, and are able to provide important information in the emerging field of in-cell NMR.

\section{ASSOCIATED CONTENT}

Materials and methods and supplementary figures. This material is available free of charge via the Internet at http://pubs.acs.org.

\section{AUTHOR INFORMATION}

\section{Corresponding Author}

*j.christodoulou@ucl.ac.uk

\section{Notes}

The authors declare no competing financial interest.

\section{ACKNOWLEDGMENTS}

We thank Dr John Kirkpatrick for valuable advice with the NMR experiments. This work was supported by the BBSRC (C.A.W., J.C.), the Wellcome Trust (C.M.D.) and HFSP (J.C.). M.D.M. and L.F.G. wish to thank the UK EPSRC for funding under Grant EP/F047991/1.

\section{ABBREVIATIONS}

$\alpha$ Syn, $\alpha$-synuclein; STE, stimulated echo.

\section{REFERENCES}

(1) Serber, Z.; Keatinge-Clay, A. T.; Ledwidge, R.; Kelly, A. E.; Miller, S. M.; Dötsch, V. J Am Chem Soc 2001, 123, 2446-2447.

(2) Sakakibara, D.; Sasaki, A.; Ikeya, T.; Hamatsu, J.; Hanashima, T.; Mishima, M.; Yoshimasu, M.; Hayashi, N.; Mikawa, T.; Wälchli, M.; Smith, B. O.; Shirakawa, M.; Güntert, P.; Ito, Y. Nature 2009, 458, 102-105.

(3) Barnes, C. O.; Pielak, G. J. Proteins 2011, 79, 347-351.

(4) Callaghan, P. T. Translational Dynamics and Magnetic Resonance: Principles of Pulsed Gradient Spin Echo NMR; Oxford University Press, 2011.

(5) Li, C.; Wang, Y.; Pielak, G. J. J Phys Chem B 2009, 113, 13390-13392.

(6) Rajagopalan, S.; Chow, C.; Raghunathan, V.; Fry, C. G.; Cavagnero, S. J Biomol NMR 2004, 29, 505-516.

(7) Verkman, A. S. Meth Enzymol 2003, 360, 635-648.

(8) Bacia, K.; Kim, S. A.; Schwille, P. Nat Methods 2006, 3, 83-89.

(9) Dix, J. A.; Verkman, A. S. Annual review of biophysics 2008, 37, 247-263.

(10) Zimmerman, S. B.; Trach, S. O. J Mol Biol 1991, 222, 599-620.

(11) Elowitz, M. B.; Surette, M. G.; Wolf, P. E.; Stock, J. B.; Leibler, S. J Bacteriol 1999, 181, 197-203.

(12) Li, C.; Charlton, L. M.; Lakkavaram, A.; Seagle, C.; Wang, G.; Young, G. B.; Macdonald, J. M.; Pielak, G. J. J Am Chem Soc 2008, 130, 6310-6311.

(13) Ferrage, F.; Zoonens, M.; Warschawski, D. E.; Popot, J.L.; Bodenhausen, G. J Am Chem Soc 2003, 125, 25412545.

(14) Wu, D.; Chen, A.; Johnson, C. J Magn Reson Ser A 1995, $115,260-264$.

(15) Augustyniak, R.; Ferrage, F.; Paquin, R.; Lequin, O.; Bodenhausen, G. J Biomol NMR 2011, 50, 209-218.

(16) Dedmon, M. M.; Lindorff-Larsen, K.; Christodoulou, J.; Vendruscolo, M.; Dobson, C. M. J Am Chem Soc 2005, 127, 476-477.
C. M. Biomol NMR Assign 2009, 3, 29-31.

Barnes, C. O.; Monteith, W. B.; Pielak, G. J. Chembiochem 2011, 12, 390-391.

Tugarinov, V.; Hwang, P. M.; Ollerenshaw, J. E.; Kay, L. E. J Am Chem Soc 2003, 125, 10420-10428.

Didenko, T.; Boelens, R.; Rüdiger, S. G. D. Protein Eng Des Sel 2011, 24, 99-103.

Ming, D.; Brüschweiler, R. J Biomol NMR 2004, 29, 363 368 .

Waudby, C. A.; Christodoulou, J. J Magn Reson 2011, 211, 67-73.

Kuchel, P. W.; Coy, A.; Stilbs, P. Magn Reson Med 1997, 37, 637-643.

Mitra, P.; Halperin, B. J Magn Reson Ser A 1995, 113, 94-101.

Selenko, P.; Wagner, G. J Struct Biol 2007, 158, 244-253. Inomata, K.; Ohno, A.; Tochio, H.; Isogai, S.; Tenno, T.; Nakase, I.; Takeuchi, T.; Futaki, S.; Ito, Y.; Hiroaki, H.; Shirakawa, M. Nature 2009, 458, 106-U11.

Baldwin, A. J.; Anthony-Cahill, S. J.; Knowles, T. P. J.; Lippens, G.; Christodoulou, J.; Barker, P. D.; Dobson, C. M. Angew Chem Int Edit 2008, 47, 3385-3387.

Waudby, C. A.; Knowles, T. P. J.; Devlin, G. L.; Skepper, J. N.; Ecroyd, H.; Carver, J. A.; Welland, M. E.; Christodoulou, J.; Dobson, C. M.; Meehan, S. Biophys J 2010, 98, 843-851.

Christodoulou, J.; Larsson, G.; Fucini, P.; Connell, S. R.; Pertinhez, T. A.; Hanson, C. L.; Redfield, C.; Nierhaus, K. H.; Robinson, C. V.; Schleucher, J.; Dobson, C. M. Proc Natl Acad Sci USA 2004, 101, 10949-10954. 
Graphical abstract:



Slow and restricted protein diffusion within the cell

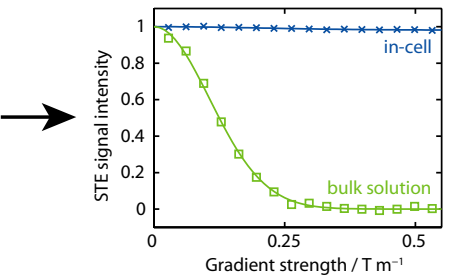

Distinction of intracellular and extracellular species using NMR diffusion measurements 


\section{Supporting Information: Rapid distinction of intracellular and extracellular proteins using NMR diffusion measurements}

Christopher A. Waudby ${ }^{1}$, Mick D. Mantle ${ }^{2}$, Lisa D. Cabrita ${ }^{1}$, Lynn F. Gladden ${ }^{2}$, Christopher M. Dobson $^{3}$ and John Christodoulou ${ }^{1}$

${ }^{1}$ Institute of Structural and Molecular Biology, University College London and Birkbeck College, Gower Street, London WC1E 6BT, UK

${ }^{2}$ Department of Chemical Engineering and Biotechnology, University of Cambridge, Pembroke Street, Cambridge CB2 3RA, UK

${ }^{3}$ Department of Chemistry, University of Cambridge, Lensfield Road, Cambridge CB2 1EW, UK

\section{Materials and Methods}

\section{Sample preparation}

The preparation of NMR samples of cells in which $\alpha$ Syn, TTHA or ddFLN5 had been expressed followed previously described protocols ${ }^{1} .100 \mathrm{~mL}$ of LB was inoculated with BL21 (DE3) Gold cells (Stratagene) and incubated at $310 \mathrm{~K}$ with shaking $\left(200 \mathrm{rpm}\right.$ ) until an $\mathrm{OD}_{600}$ of 0.6. Cells were harvested by centrifugation $(1800 \times \mathrm{g}, 15 \mathrm{~min}, 277 \mathrm{~K})$ and were resuspended in $50 \mathrm{~mL}$ of M9 minimal media containing $0.1 \%(\mathrm{w} / \mathrm{v}){ }^{15} \mathrm{NH}_{4} \mathrm{Cl}$ as the sole nitrogen source. After incubating for a $10 \mathrm{~min}$ recovery period at $310 \mathrm{~K}$ with shaking (200 rpm), expression was induced with $1 \mathrm{mM}$ IPTG for 4 hrs. Cells were then harvested by centrifugation (1800 x g, $15 \mathrm{~min}, 277 \mathrm{~K}$ ), resuspended in 50 $\mathrm{mL}$ of $\mathrm{M} 9$ minimal media containing unlabelled $\mathrm{NH}_{4} \mathrm{Cl}$ to reduce background labelling, then harvested again by centrifugation. The cell pellet was resuspended as a $40 \%(\mathrm{v} / \mathrm{v})$ slurry in unlabelled M9 media containing $10 \% \mathrm{D}_{2} \mathrm{O}$ and $0.001 \% \mathrm{DSS}$, and $600 \mu \mathrm{L}$ was transferred into a preshimmed NMR tube.

Lysed samples were prepared from NMR samples for which the initial cell integrity had been verified, by the methods described in this work, to be at least $98 \%$. Samples were lysed by plunging the sample in a $1.5 \mathrm{~mL}$ Eppendorf tube into liquid $\mathrm{N}_{2}$ and subsequently thawing at room temperature. Following NMR observations the sample was centrifuged (2000 x $g, 10 \mathrm{~min}, 277 \mathrm{~K}$ ), and the supernatant was removed for repeated observation by NMR, to determine the extent of lysis.

ddFLN5, with selective ${ }^{13} \mathrm{CH}_{3}$ labeling of the isoleucine amino acid residues, was produced in BL21 (DE3) Gold E. coli cells (Stratagene). Cells were initially adapted in $99.9 \%{ }^{2} \mathrm{H}_{2} \mathrm{O}$ M9 minimal media containing ${ }^{12} \mathrm{C}$ - $\mathrm{d}_{7}$-glucose, using a stepwise approach in order to maintain the growth kinetics of $E$. coli. The resulting culture was used to inoculate $100 \mathrm{~mL}$ of $99.9 \%{ }^{2} \mathrm{H}_{2} \mathrm{O}$ M9 
minimal media with ${ }^{12} \mathrm{C}-\mathrm{d}_{7}$-glucose $(2 \mathrm{~g} / \mathrm{L})$ as the sole carbon source, and the cells were incubated at $37^{\circ} \mathrm{C}$. At mid-log phase, $8 \mathrm{mg}$ of the isoleucine precursor, 2-ketobutyric acid-4- ${ }^{13} \mathrm{C}-3,3-\mathrm{d}_{2}$ was added and the cells were allowed to recover for one hour. Following induction with $1 \mathrm{mM} \mathrm{IPTG,}$ expression proceeded for 4 hours and the cells were then prepared immediately for NMR analysis as for $\alpha$ Syn. Following NMR observations, cells were harvested and lysed by sonication. ddFLN5, containing a hexa-histidine tag, was then purified by immobilized metal affinity chromatography using standard protocols. Briefly, the lysate was bound to Ni-NTA agarose (Qiagen), washed with $25 \mathrm{mM}$ imidazole, and eluted with $250 \mathrm{mM}$ imidazole before dialysis and storage in $\mathrm{H} 2 \mathrm{O}$ at $-80^{\circ} \mathrm{C}$.

\section{NMR spectroscopy}

NMR data were acquired at 16.4 $\mathrm{T}$ on a Bruker Avance III spectrometer equipped with a TXI cryoprobe, with a unidirectional gradient coil generating a maximum gradient of $0.55 \mathrm{~T} \mathrm{~m}^{-1} .{ }^{15} \mathrm{~N}$ edited diffusion NMR experiments were measured at $310 \mathrm{~K}$ using a $300 \mathrm{~ms}{ }^{1} \mathrm{H}$ stimulated echo with storage as longitudinal magnetisation on ${ }^{15} \mathrm{~N}$ during the diffusion delay ${ }^{2}$. Bipolar trapezoidal gradient pulses (shape factor $s=0.9$ ) were applied with a total pulse length, $\delta$, of $4 \mathrm{~ms}$ and a spacing, $\tau$, of $200 \mu \mathrm{s}$. Echos were acquired in 16 scans at 16 gradient strengths ranging linearly from 5 to $95 \%$ of the maximum gradient, with a total acquisition time of 6 min. ${ }^{13} \mathrm{C}$-edited diffusion measurements ${ }^{3}$ were acquired using a $300 \mathrm{~ms}$ stimulated echo and bipolar trapezoidal gradient pulses with a total length, $\delta$, of $4 \mathrm{~ms}$ and a spacing, $\tau$, of $200 \mu \mathrm{s}$. Echos were acquired in 64 scans at 8 gradient strengths ranging linearly from 5 to $95 \%$ of the maximum gradient, with a total acquisition time of $12 \mathrm{~min}$.

NMR data were processed in nmrPipe ${ }^{4}$ with a linear baseline, and the entire amide envelope was extracted and integrated. Data were fitted to the Stejskal-Tanner equation (Eq. 1, main text) or, for the analysis of partially lysed samples, a two-component model representing the attenuation due to diffusion of extracellular species, and a constant term representing intracellular species, the intensity of which did not decay with the gradient strengths used here:

$$
I(G)=I_{\text {extracellular }} \exp \left[-\gamma^{2} G^{2} \delta^{2} s^{2}(\Delta-\delta / 3-\tau / 2) D_{\text {app }}\right]+I_{\text {intracellular }}
$$

Diffusion-edited ${ }^{1} \mathrm{H}^{-15} \mathrm{~N}$ HSQC spectra (using the XSTE-3D experiment ${ }^{5}$, adapted for onedimensional gradient probes) were acquired at $277 \mathrm{~K}$ with a $300 \mathrm{~ms}$ stimulated echo and bipolar trapezoidal gradient pulses of length, $\delta$, of $4 \mathrm{~ms}$. Two spectra were acquired, in an interleaved manner, having gradient strengths of 5 and $95 \%$ of the maximum. Diffusion-edited ${ }^{1} \mathrm{H}_{-}{ }^{13} \mathrm{C}$ HMQC spectra $^{3}$ were also acquired using a $300 \mathrm{~ms}$ stimulated echo and bipolar trapezoidal gradient pulses 
with a total length $\delta$ of $4 \mathrm{~ms}$, with gradient strengths 5 and $95 \%$ of the maximum. ${ }^{1} \mathrm{H}_{-}{ }^{13} \mathrm{C}$ HMQC spectra of purified ddFLN5 were acquired using identical parameters.

NMR experiments utilizing high gradient strengths were acquired at $7.1 \mathrm{~T}$ on a Bruker DMX spectrometer equipped with a Diff30 probe, which provided a maximum gradient strength of $11.7 \mathrm{~T}$ $\mathrm{m}^{-1}$. All measurements were performed at $310 \mathrm{~K}$, using ${ }^{1} \mathrm{H}$ spin-echos with trapezoidal gradients having a $0.1 \mathrm{~ms}$ ramp time. A comparison with uninduced cells showed that the methyl resonance at $0.7 \mathrm{ppm}$ provided excellent selectivity for $\alpha$ Syn, hence this signal was integrated for further analysis. Spectra were individually phased and baseline corrected. ${ }^{1} \mathrm{H}$ spin-echo experiments were acquired with a gradient pulse length of $2 \mathrm{~ms}$ and the diffusion time, $\Delta$, varied from 11 to $20 \mathrm{~ms}$. Each experiment was recorded at 16 linearly-spaced gradient strengths, with 32 scans acquired at each gradient point, and data were fitted to Eq. 1 (with $\tau=0$ as bipolar gradients were not used) to determine the apparent RMS displacement, $Z_{\mathrm{rms}}=\left(2 D_{\mathrm{app}} \Delta\right)^{1 / 2}$.

\section{Brownian dynamics simulations}

Monte Carlo simulations of diffusion were performed as previously described ${ }^{6}$, using a standard geometry for an E. coli cell, i.e. a cylinder with hemispherical caps at both ends having maximum dimensions of $0.5 \times 0.5 \times 2 \mu \mathrm{m}$.

\section{References}

(1) Serber, Z.; Selenko, P.; Hänsel, R.; Reckel, S.; Löhr, F.; Ferrell, J. E.; Wagner, G.; Dötsch, V. Nat Protoc 2006, 1, 2701-2709.

(2) Ferrage, F.; Zoonens, M.; Warschawski, D. E.; Popot, J.-L.; Bodenhausen, G. J Am Chem Soc 2003, 125, 2541-2545.

(3) Didenko, T.; Boelens, R.; Rüdiger, S. G. D. Protein Eng Des Sel 2011, 24, 99-103.

(4) Delaglio, F.; Grzesiek, S.; Vuister, G.; Zhu, G.; Pfeifer, J.; Bax, A. J Biomol NMR 1995, 6, 277-293.

(5) Augustyniak, R.; Ferrage, F.; Paquin, R.; Lequin, O.; Bodenhausen, G. J Biomol NMR 2011, 50, 209-218.

(6) Waudby, C. A.; Christodoulou, J. J Magn Reson 2011, 211, 67-73. 


\section{Supplementary Figures}

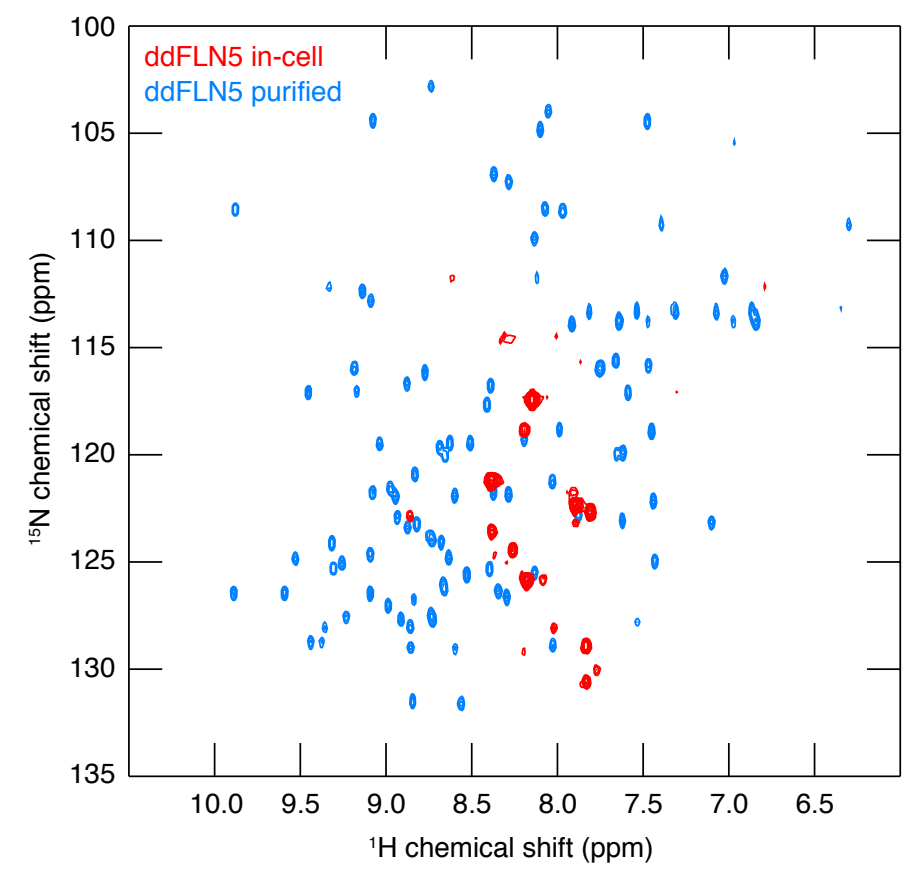

Figure S1. ${ }^{1} \mathrm{H}^{15}{ }^{15}$ HSQC NMR spectra of E. coli cells in which ddFLN5 had been expressed (red), and purified ddFLN5 in bulk solution (blue).

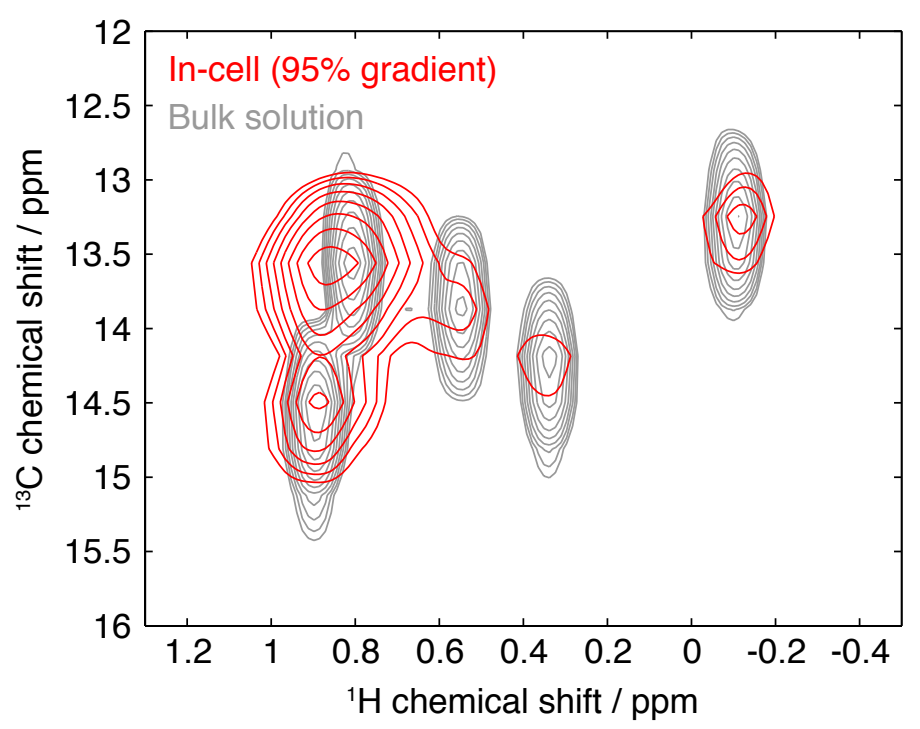

Figure S2. Diffusion-edited (300 ms STE) ${ }^{1} \mathrm{H}_{-}{ }^{13} \mathrm{C}$ HMQC NMR spectrum of $\left[\mathrm{Ile}-{ }^{13} \mathrm{CH}_{3}\right]$-ddFLN5 expressed within $E$. coli cells (red), at the maximum accessible gradient strength $\left(0.53 \mathrm{~T} \mathrm{~m}^{-1}\right)$, and the ${ }^{1} \mathrm{H}-{ }^{13} \mathrm{C}$ HMQC NMR spectrum of purified [Ile- ${ }^{13} \mathrm{CH}_{3}$ ]-ddFLN5 in bulk solution (grey). 


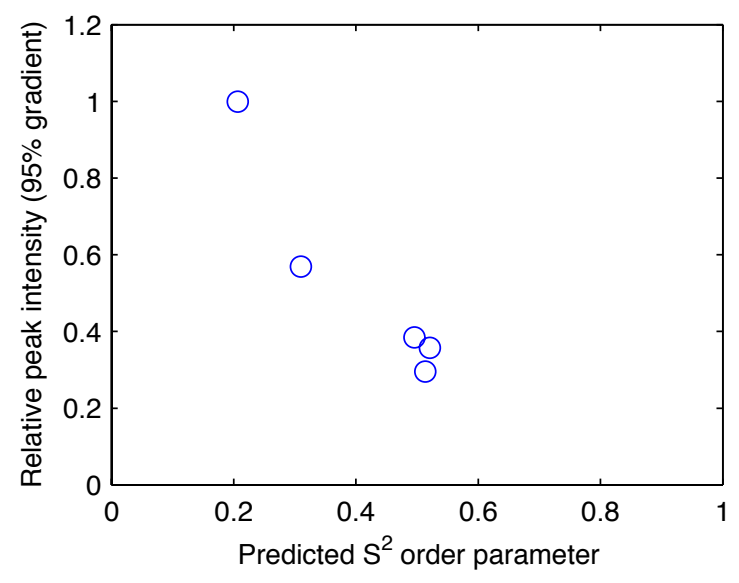

Figure S3. Inverse correlation $\left(r^{2}=0.90\right)$ of intracellular ddFLN5 diffusion-edited HMQC peak intensities $v s$ isoleucine methyl sidechain $S^{2}$ order parameters predicted from the crystal structure (pdb 1QF8) (ref. 21). 